\title{
Cognitive Architecture to Analyze the Effect of Intrinsic Motivation with Metacognition over Extrinsic Motivation on Swarm Agents
}

\author{
Ashwini Kodipalli \\ Department of Computer Science and Engineering, Global Academy of Technology, India
}

\begin{abstract}
Article Info
Article history:

Received Dec 28, 2017

Revised Mar 23, 2018

Accepted Jul 5, 2018

Keyword:

Agents

Cognitive architecture

Intrinsic motivation

Metacognition

Swarm intelligence

ABSTRACT

This research work describes the setup of framework for testing the performance of intrinsically motivated swarm agents over extrinsic motivation. The performance is tested through the simulation. The result demonstrates that agents with intrinsic motivation for specific goal have high metacognitive ability. It also shows group performance of agents with metacognitive ability is better than the group of agents with extrinsic motivation exhibiting cognitive ability. Goal setting theory of motivation is applied to the group of agents in order to analyse the intelligent behaviour of the agents. This research is mainly focusing on why and how group performance by swarm agents is better than individuals. This approach requires design of ambient testbed where swarm agents demonstrate cognitive actions to metacognitive actions. This research is aiming to prove that group performance by swarm agents is higher due to type of agents chosen with intrinsic motivation and thus proves intrinsic motivation is better than extrinsic motivation. Agent behaviour in a group can be analysed using different metrics like resource collection, life expectancy, level of motivation and task assigned.
\end{abstract}

Copyright () 2018 Institute of Advanced Engineering and Science. All rights reserved.

\section{Corresponding Author:}

Ashwini Kodipalli,

Department of Computer Science and Engineering,

Global Academy of Technology,

Bangalore, India.

Email: ashwini.kodipalli@gmail.com

\section{INTRODUCTION}

Motivation is a forces acting internally or externally on or within a person that makes the person goal oriented or put voluntary efforts to achieve the goal. Motivation is the energy to the behavior of the person in the work. According to the Richard M Ryan, Edward L Deci [1] there are two types of motivation: Intrinsic and Extrinsic.

Intrinsic motivation is the self-desire to achieve something, to analyze one's capacity, to observe and gain knowledge. Motivation that depends on internal factors is called intrinsic motivation. According to the Dev [2] intrinsic motivation is defined participating in the activity based on the curiosity, desire to engage for the sake of participation and completing the task, desire to contribute. According to Regina M Shia [3] people with intrinsic motivation has goal to learn and goal to achieve. Internal factors can be the satisfaction of accomplishing something or the desire to do work. Intrinsic is feeling positive internally which causes the motivation. This is due to interest or enjoyment of the task. There is no desire for the reward. Motivation that depends on external factors is called extrinsic motivation. According to the Hoyenga and Hoyenga [4], extrinsic motivation refers to motivate that are outside of and separate from the behaviors they cause. Performance of the activity mainly depends in order to attain a desired outcome. Extrinsic is wanted to get the specific result or the outcome. External factors can be money. Deci stated that money which is an external 
factor has a negative effect on behavior. He also told that if a person does task based on these external factors, he will be less motivated towards the task. If a person is not externally motivated, he would be in control and will be intrinsically motivated. External factors like money reduce intrinsic motivation.

\section{PERFORMANCE AND MOTIVATION RELATIONSHIP}

Performance and the motivation are two different factors. First the motivation act as an internal drive for an individual to achieve the goal. They can be influenced by the other factors. The motivated people when they complete the task, they will have a satisfaction after completing their task. Some people get motivated based on the external values. When an individual is externally motivated, they look what they get after they reach their goal. Based on the situations, the performance can be measured in the different ways like satisfactory, unsatisfactory, good, bad, high or low and it depends on the individual tasks. As per the managers it is being measured as being high, average or low performance. Hence the individual has to be well motivated to complete their task at high levels. The managers know that when the employees are motivated, they perform in high level. Also when they are motivated from their job, they tend to increase the job satisfaction. When the employee has their satisfaction in the job, then they tend to get committed to their organization when compared to the other people in the organization. Hence the motivated person becomes beneficial to the organization.

Various researches have been conducted to relate both the motivation and the performance to see, what motivates the individual and the group to perform at high level. A particular study on fix salary pay verses the performance was conducted on motivated individuals by Cadsby, Song and Tapon in the year 2007. The pay for the individuals was based on their performance and whereas the fixed pay was not affecting the individual performance.

In the study eight participants were involved. Each participant was given the individual tasks to perform. The people were randomly assigned for the groups of pay on performance and the fixed salary. The people were given an option of changing the group. The results were, the group where the individuals were paid based on the performance, their performances was high when it is compared to the other group of fixed pay. With this study, it is clear that the individuals were more motivated to perform at the high level when they were paid more based on their performance [5].

Clasen, Silva-Jalonen \& Butler [6] conducted an experiment, where promise of rewards has an impact on individual performance. The individuals were asked to divide into groups and informed them that the feedback on their work is given to the individuals who are performing in the group. The result was absorbed that, when an individual were told that the feedback will be given to their work, then they were more motivated and performed the task in a better way. Hence the individuals will be more motivated when they know that there task will be observed and the feedback is given, they are more motivated and they tends to give high performance.

Hence based on these two studies on individual performance and motivation, performance and motivation are directly related [6]. In 2005, Wegge and Haslam conducted an experiment on group in contrast to individuals. There were four groups told to do their best. All four groups finished their task. The performance and the motivation were well demonstrated in this experiment. It was been demonstrated that the group performance was high when the group had a specific goal to perform and observed that their performance was high when compared with the group where they were told to do your best. The group's goal setting also increases the individual performance. It motivates them to achieve the goal. Therefore the performance is more when the group has a well desired goal setting [7].

In 1998, Vegt and Vliert conducted a test that examined interdependent in work team and how it affected individual's motivation and the performance. Task interdependent is when one group member depends on the group member to complete the task. The outcome of interdepend is, group members believes that the outcome of a task depends on the successful goal attainment. In this the result was found that the individual was more responsible of their work more as well as team member work overall interdependence was high. The individuals were more motivated to increase the performance of other people. Also when they are working in group and when the group had a goal, the individual performance is high.

It is known that the individual performance will be high when they have goal and also in the group when they have a common goal they will be motivated to work and hence the performance increases. People are more motivated when they work in group than in the individual task. The research says that the dependent variables are the individual level of performance when they are doing their task and their motivation level. Performance in the group will be at the high levels compared to the individual task [8]. 


\section{NEWLY PROPOSED SACA (SWARM AMBIENT COGNITIVE ARCHITECTURE)}

SACA is a 3-column and four layered architecture as shown in the Figure 1 proposed for demonstrating agents with the intrinsic motivation for the goal will always perform than the agents with extrinsic motivation. And also performance of the group will be better if a group has agents with intrinsic motivation. This proposed architecture is influenced by SMCA [9], EM-One [10] and CRIBB [11]. The layers of this architecture have different agents with different level of motivation that shows different level of capabilities.

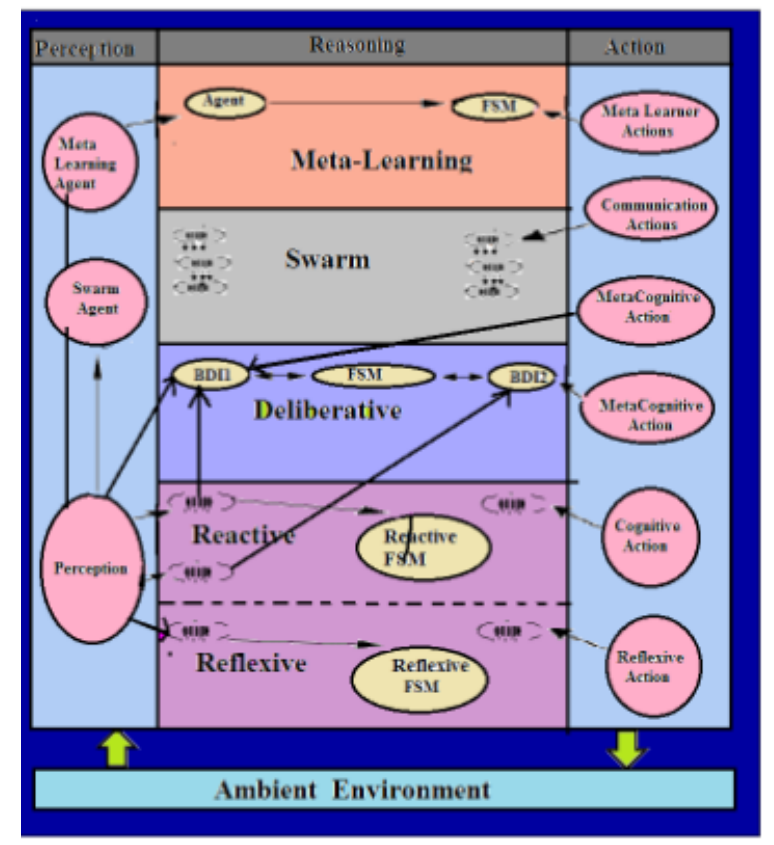

Figure 1. SACA architecture

The different swarm agents are reflexive, reactive and deliberative. The first level at the lower side exhibits simple reflexes such as moving towards the corners of the environment without any goal oriented; avoid hitting the wall and other agents. Hence there is no motivation towards the goal. At the upper level in the first layer is highly reactive to the goal for the reward. Reward defined here is reaching to the jewel point. Since it is highly goal oriented for reward, we can see extrinsic motivation in this agent. At the second level is BDI (Belief, Desire and Intension) layer which builds a desire, intension set for agent drives the agent. This agent works at metacognitive strategies. This agent can sense its internal state before performing the task and act accordingly in the environment. This agent has intrinsic level of motivation. At the third level the highly motivated agents communicate with each other and perform the task faster. At the higher level the learning takes place through q-learning process and CST (Constructive Skill Tree) method.

The ambient testbed is implemented using SWI-Prolog 6.6.4.The Ambient testbed experiments include cognitive and psychological aspects on the architecture. The different dynamic parameters are food, diamonds and agents as shown in the Figure 2. Food and diamond are agent's energy and performance parameters. Agents are created using prolog graphics. All the three parameters are dynamic because they are randomly created in the environment and their location changes for every new start of the experiment. Based on the experimental requirements the parameters can be changed and is defined in the configuration module. The agent can differentiate between the parameters and is consumed based on the requirements. Number of resource parameters needed is selected using the sliding bar, the initial energy for an agent and the number of cycles the agent has to perform. Food is considered as nutrition for an agent. In the environment the food is green small square shaped parameter. Diamond is considered as a performance parameter. Collecting the diamonds is the main aim of the agents in the experiment. Diamond is small white circle shaped parameter. An agent is an actor. An agent senses the environment through sensors and acts to its environment through actuators. In the environment the agents are big circle of different colors. 


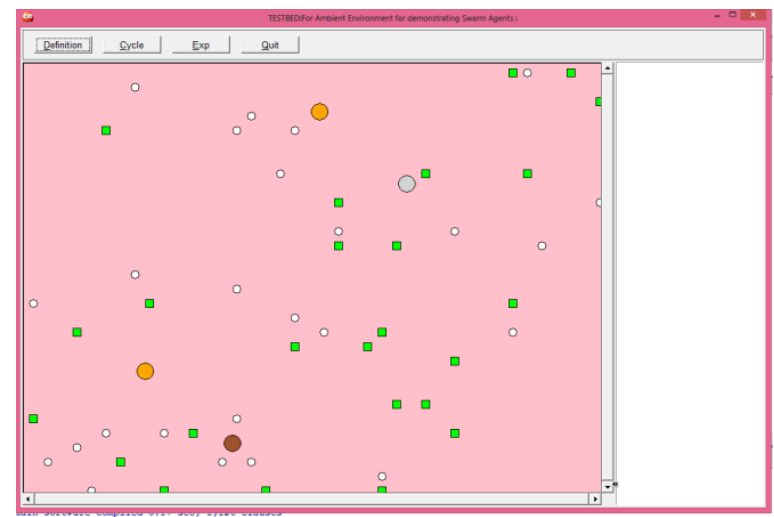

Figure 2. Ambient testbed for the swarm agents

The working of the agents is represented by structural diagram as shown in the Figure 3. The swarm agents are created and are stored in the agent's database. All agents stored in the database are placed in the environment. The two different parameters are created and are stored in the parameter database. These parameters are placed in the environment. Agents and parameters are placed dynamically in the environment. The swarm agents sense the parameters such as food and diamond in the environment. The agent has a perceptual range of 5 steps and can see all the parameters at its exposure. The sensed information by the agent are stored in the memory. Further the sensed data is sent for reasoning where decision making takes place. Based on the decision appropriate action is taken. The action can be any of the following based on the properties defined for the agent.

a. Reflexive agent collects diamond and consumes the food on its way to the edges or center of the environment.

b. Reactive agent collects only diamond.

c. Deliberative agent collects diamond but to manage its internal energy, the agent collects the food.

Once the parameters are collected, the parameters are removed from the environment and database. For every move the agent loses 2 units of energy. On reaching the energy to zero the agent will die from the environment and removed from the database.

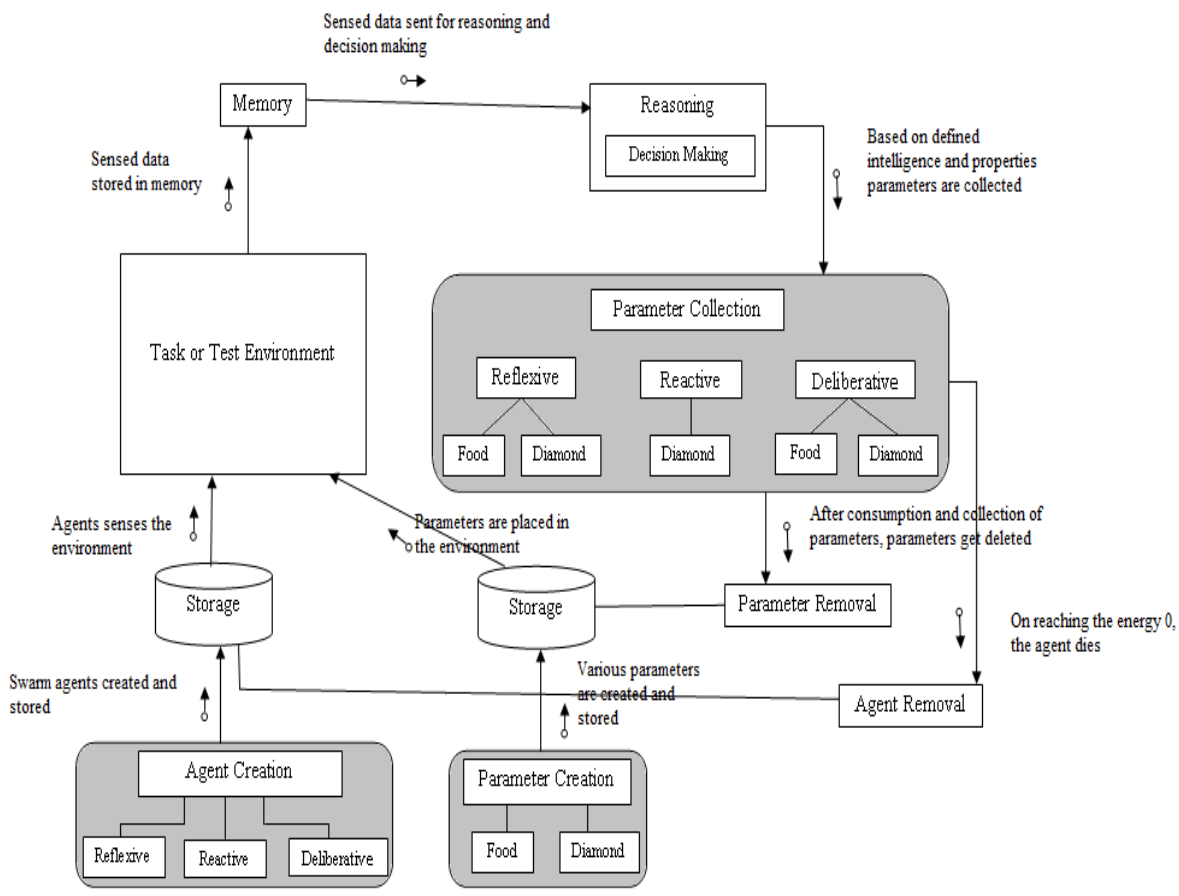

Figure 3. Structural diagram 


\section{RESULTS}

SACA architecture is designed to check how individual agents will behave in a group, how agents behavior will have on impact on the group performance. Agent behavior can be analyzed using different metrics like competition, life expectancy and the social interaction with respect to environment and its parameter. The simulation demonstrates the complex interaction between different type of agents, agents behavior with respect to use of energy and time to make decisions.

\subsection{Study one: Comparing the performances of the swarm agents}

Comparison among the agents is very important to find out who among the agents are very efficient and finding out the characteristic of that particular agent. This helps in framing the efficient group and hence the performance of the group can be improved. In comparison with all three types of agents, Deliberative agents are highly intrinsically motivated, Reactive agent is extrinsically motivated and Reflexive agent is less/0 motivated.

This result shows that the deliberative agent can reason about their change of aims, sense their state and achieve their goals. Thus exhibiting decision making and intelligent behaviors. Decision making level in the experiment is switching to food when it is hungry i.e. when its energy decreases below to threshold value and when it has normal energy, it switches to goal (collection of diamonds) demonstrate psychological and intelligent behavior. Deliberative agents are more efficient in managing their energy level collecting more goals. Cognition agents like reactive and reflexive agents cannot control their energy levels and sometimes falls to zero energy level before completing the maximum cycles. Deliberate agent collected more number of goals then the cognitive agents.

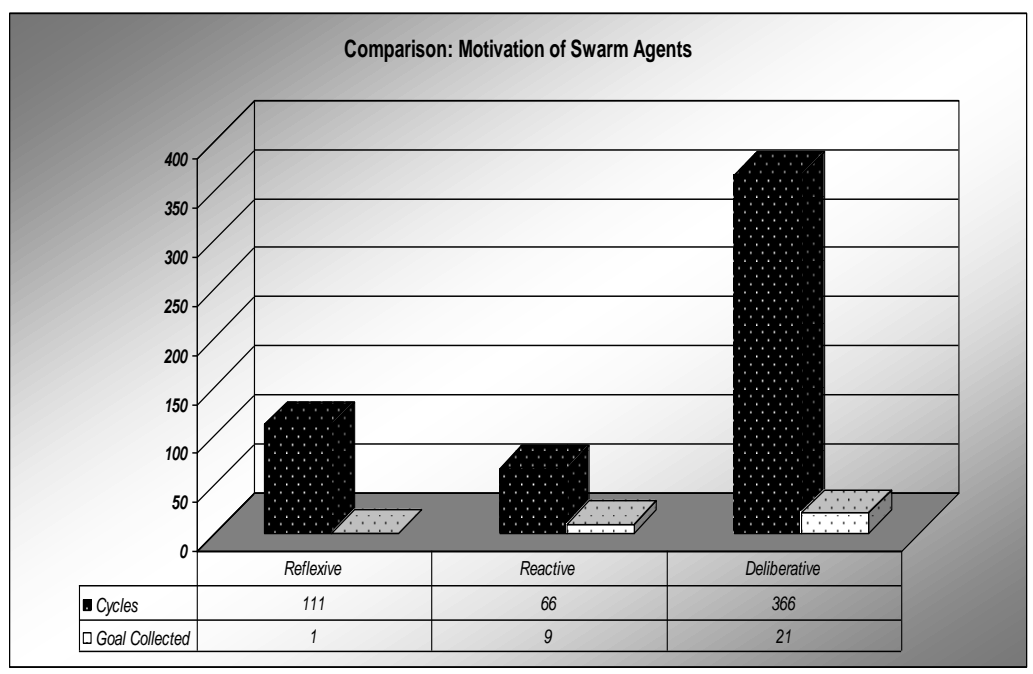

Graph 1. Comparison of swarm agents

This concludes that agent with intrinsic motivation performance is much higher than the agent with the extrinsic motivation and zero motivation agent. Intrinsic motivation is better than the extrinsic motivation because intrinsic motivation plays a major role in human's life activities. Before learning takes place, in learning process, the learners should be active and learning will be optimal when learners were internally willing to be involved or when they are interested volitional [12].

Deliberative agent has complex intelligent behaviors. Deliberative agents has complete control in managing food and goal, try to balance motivations. Deliberative agents collect more goals and manage higher life expediency than other agents. In this results process that deliberative agent has more control and self-reflection catalyst. Thus stating that deliberative agent this highly motivated than other agents and improve the performance. Agents with intrinsic motivation use metacognitive strategies and thus becomes flexible, effective and independent learners. Metacognitive strategies were found to be strongly related to performance and intrinsic motivation [13]. 


\subsection{Study two: Analysis of the group performance}

The following graph is generated to check how performance of agents in a group varies with proper motivation and coordination. Since group of agents are performing together, always group performance is greater than an individual performance. With better group members, performance of the group will be better. What makes the individual member better? This experiment provides the solution to the question, group performance is always better than individual performance. From the Graph 1, it is clear that deliberative agent is highly motivated towards the goal because it is intrinsically motivated to perform the task. In the group formation, it has to be considered so that the group performance should be higher. So it is important to be careful in framing the group. Since deliberative agent is highly motivated to the goal, the group should be the agents with the highly motivated towards the goal. So this experiment to analyses the performance of agents in a group by considering the group with two deliberative agents, reactive and reflexive agents. So intrinsic motivated agent 1 and agent 2 is considered. It is observed that both agents compete with each other for performing, they both motivate each other by surviving almost equal number of cycles and time taken to complete the task is also faster when the group has the homogeneity in agents. Reactive agent is extrinsically motivated towards the goal and the reflexive agent is zero motivated towards the goal. The graph shows that agent1 has survived for 221 cycles out of 500 and agent 2 also for 221 cycles. Agent 1 has collected 12 diamonds out of 25 and agent 2 collected 13. From Graph 2 it is shown that deliberative agent alone collected 21 diamonds. But in the group the agents collected 25 out of 25 diamonds. This satisfies the property of "Synergy" which means collective performance must produce better results than the sum of individual performance. According to the social research, motivational gain is achieved from this experiment.

After the experiment the results concludes that extrinsically motivated agent has collected 7 diamonds and survived for the 51 cycles and the zero motivated agent has survived for 66 cycles but not collected any diamonds. With this result it shows that if a group has this combination of the agents then it leads to motivational loses where if one person is performing the task the other will let the person to perform and the person will relax without performing any task.

The graph demonstrates that motivation of an individual is essential for successful motivation of the group which means when all agents in a group are highly motivated for a specific goal or a challenging goal, then competition for a goal to complete the task among the motivated agents increases and thus the performance of the group will be effective.

If the agents are less motivated to the specific goal in a group, then the performance of the group declines and leads to the motivational losses like social loafing. Thus performance of the group depends not only on the communication among the agents but also on the specific goal, the motivational levels of individual agents in a group (intrinsic or extrinsic), composition of the group (homogeneity in members of the group), cohesiveness (commitment towards staying together in a group).

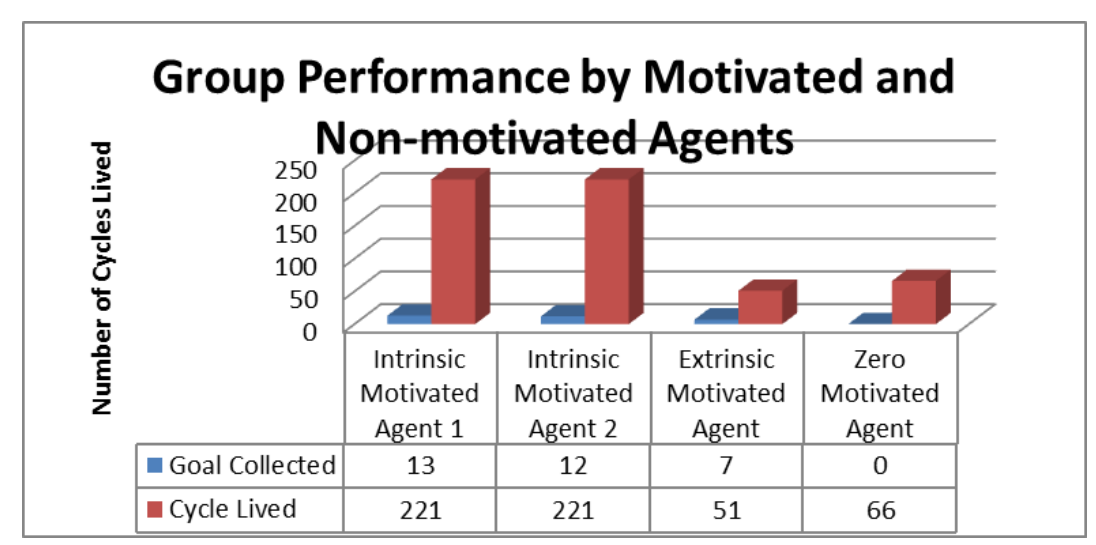

Graph 2. Analysis of agents in a group

\section{CONCLUSION}

In SACA architecture, group of agents works for the same tasks. It was been demonstrated in SACA that the group performance was high when they had a specific goal and their performance was high. The group's goal setting also increases the individual performance in SACA. It motivates agents to achieve the goal. Performance of swarm agents in SACA is high, because individuals are more responsible of their task when they work in a group. Therefore the performance is more when they have a well desired goal setting with highly motivated agents. SACA model gives the clearly demonstrates that communication alone among 
the agents is not sufficient for the performance to increase but even on the type of the task (specific goal), the motivational levels of individual agents in a group, composition of the group(type of individuals in the group). Therefore agents with the intrinsic motivation perform better than the agents with the extrinsic motivation.

SACA architecture is developed from the inspiration of natural swarms. Hence communication is an important aspect in the proposed architecture. A low level communication is implemented among the group of agents in SACA. In SACA architecture, group of agents works for the same tasks. It was been demonstrated in SACA that the group performance was high when they had a specific goal and their performance was high. The group's goal setting also increases the individual performance in SACA. It motivates agents to achieve the goal. Therefore the performance is more when they have a well desired goal setting with all agents highly motivated for the goal. Performance in swarm agents in SACA is high, because individuals are more responsible of their task when they work in a group. It is known that the individual performance will be high when they have goal and also in the group when they have a common goal they'll be motivated to work and hence the performance increases.

Comparing to the existing cognitive architectures like EM-ONE, CRIBB and SMCA, SACA architecture is a cognitive architecture, where group of agents communicate with each other and motivates each other to perform for a common task. SACA model gives the clearly demonstrates that communication alone among the agents is not sufficient for the performance to increase but even a motivational factor among the agents adds to the performance.

\section{REFERENCES}

[1] Ryan, et al., "Self-determination Theory and Facilitation of Intrinsic Motivation, Social Development, and wellbeing", American Psychologist, vol. 55, no. 1, pp. 68-78, 2000.

[2] P. C. Dev, "Intrinsic Motivation and Academic Achievement", Remedial and Special Education, vol. 18, no. 1, pp. 12-19, 1997.

[3] R. Shia, "Academic Intrinsic and Extrinsic Motivation and Metacognition", Assessing academic intrinsic motivation: A look at student goals and personal strategy, Wheeling Jesuit University, 2005, http://www.cet.edu/research/pdf/motivation.pdf.

[4] K. B. Hoyenga and K. T. Hoyenga, "Motivational Explainations of Behavior", Monterey, CA, Brookes/Coles Publishing Company, 1984.

[5] C. B. Cadsby, et al., "Sorting and Incentive Effects of Pay for Performance: An Experimental Investigation", Academy of Management Journal, vol. 50, no. 2, pp. 387-405, 2007.

[6] B. L. Cooper, et al., "Creative Performance on an in-basket Exercise: Effects of Inoculation Against Extrinsic Reward”, Journal of Managerial Psychology, vol. 14, no. 1, pp. 39-57, 1999.

[7] J. Wegge and A. Haslam, "Improving work Motivation and Performance in Brainstorming Groups: The Effects of three Group goal-setting Strategies", European Journal of Work and Organizational Psychology, vol. 14, no. 4, pp. 400-430, 2005.

[8] G. S. V. D. Vegt, et al., "Motivating Effects of Task and Outcome Interdependence in Work Teams", Group \& Organization Management, vol. 23, no. 2, pp. 124-143, 1998.

[9] M. V. Vijayakumar, "Society of Mind Approach to Cognition and Metacognition in a Cognitive Architecture", $\mathrm{PhD}$ Thesis, University of Hull, UK, 2008.

[10] P. Singh, "EM-ONE: Architecture for Reflective Commonsense Thinking", PhD Thesis, 2005, http://web.media.mit.edu/ pus.

[11] K. Bartsch and H. Wellman, "Young Children's Attribution of Action to Beliefs and Desires", Child Development, vol. 60, pp. 946-964, 1989.

[12] E. L. Deci and R. M. Ryan, "The Support of Autonomy and Control of Behaviour", Journal of Personality and Social Psychology, vol. 53, no. 6, pp. 1024-1037, 1987.

[13] P. R. Pintrich and E. V. DeGroot, "Motivational and self-regulated Learning Components of Classroom Academic Performance", Journal of Educational Psychology, vol. 82, no. 1, pp. 33-40, 1990. 